\title{
Quercetin Protects Rat L6 Myocytes from Antimycin A-Induced Mitochondrial Dysfunction
}

\section{Vijay M Kale*}

College of Pharmacy, Roseman University of Health Sciences, South Jordan, Utah

*Corresponding author: Vijay M. Kale, Assistant Professor of Pharmaceutical Sciences, College of Pharmacy, Roseman University of Health Sciences, South Jordan, Utah, Tel: 801-878-1078; E-mail: vkale@roseman.edu

Received date: December 28, 2015; Accepted date: January 25, 2016; Published date: January 28, 2016

Copyright: (c) 2016 Kale VM. This is an open-access article distributed under the terms of the Creative Commons Attribution License, which permits unrestricted use, distribution, and reproduction in any medium, provided the original author and source are credited.

\begin{abstract}
Objective: Mitochondrial dysfunction is often associated with various disorders such as diabetes, Alzheimer's etc. Reactive oxygen species (ROS), aging, and reduction of mitochondrial biogenesis contribute to mitochondrial dysfunction. Antimycin-A (AMA) damages the mitochondria through inhibition of mitochondrial electron transport. The present study sought to investigate effects of quercetin on rat L6 cells and whether quercetin protects mitochondria against oxidative damage caused by AMA.
\end{abstract}

Methods: Rat L6 myocytes were used for the study. Effects of quercetin on Antimycin-A induced mitochondrial dysfunction was studied using cytotoxicity, ATP levels, mitochondrial superoxide production and NDUFB8 mRNA expression.

Results: In this study, exposure of L6 myocytes to AMA induced an increase cell death, decreased ATP content, followed by a decrease in mitochondrial superoxide, and decreased expression of NDUFB8. We found that quercetin protected myocytes from antimycin-A (AMA) induced L6 cell death as evidenced from increased lactate dehydrogenase (LDH) leakage into extracellular medium, protected ATP production, prevented increase in oxidative stress and restored levels of NDUFB8 mRNA expression implying improved mitochondrial function.

Conclusion: These results suggest that the quercetin showed protective effect against AMA-induced mitochondrial dysfunction by increasing ATP production, decreasing oxidative stress, and restoring mitochondrial function.

Keywords: Quercetin; Mitochondrial biogenesis; Oxidative stress; Antimycin-A; Cytotoxicity; ATP; Myocytes

\section{Introduction}

Mitochondrial biogenesis is an important process responsible for regulating energy demand of the cell. Accordingly, it is affected by a variety of factors, like exercise, low temperature, and oxidative stress $[1,2]$. Mitochondrial biogenesis is a type of mitochondrial dysfunction and can play a role in many diseases, like diabetes and Alzheimer's, etc. $[3,4]$. Increased oxidative damage and inflammation can cause mitochondrial damage that may lead to serious acute and chronic pathologies such as multi organ failure, neurodegeneration, cardiovascular diseases, and cause aging. Mitochondrial biogenesis enhance cellular function and survival in-vitro and in-vivo and promote cellular recovery from damage caused by adverse environmental, pathophysiological, and/or infectious agents $[4,5]$. Mitochondrial biogenesis is a complex process that includes growth and division of pre-existing mitochondria, ultimately leading to increased energy production in cells. High energy status most likely determines the outcome of cellular insult caused by oxidative stress, chemical or mechanical injury, inflammation, and routine tissue repair [6].

Due to the large number of mitochondria myocytes play an important role in glucose uptake and burning it into ATP. Beneficial effects of caloric restriction, diet, and physical exercise have been noted in diabetes, some of these effects are attributed to mitochondrial biogenesis as newly formed mitochondria are more efficient in terms of energy utilization [7]. Number of chemicals from plant origin and plant extracts has been shown to increase mitochondrial biogenesis [3]. Polyphenols represents beneficial group of naturally occurring compounds with hypoglycemic potentials. These are the most abundant antioxidants in the human diet and are commonly found in onions, apples, tea, coffee, red wine, etc. [8]. Chemically, flavonoids can be widely classified into different categories such as flavanols, flavones, catechins, flavanones, etc. [9]. Quercetin is the most well researched of all flavonoids and it is one of the most potent scavengers of reactive oxygen species including superoxide and reactive nitrogen species [10]. Quercetin has also been reported to exhibit anti-oxidative, antitumor, anti-inflammatory, vasodilatory effects [11]. Besides ROS scavenging activity it has been shown to promote mitochondrial biogenesis and improving insulin sensitization in various in-vivo and in-vitro studies [12]. Quercetin has been shown to promote mitochondrial biogenesis in brain and muscle and improve exercise tolerance $[13,14]$.

In mitochondrial respiratory chain, Complex I and III are major sources of intracellular superoxide and oxidative stress [15]. Antimycin-A is an inhibitor of complex III. The binding of AMA to complex III is directly associated with inhibition of its enzymatic activity $[16,17]$ thus increasing ROS formation through complex I and 
II [18]. Mitochondrial biogenesis has been studied in muscle cell lines as well as in animal models, however these cells has not been used as model to screen chemicals for mitochondrial biogenesis. Thus in the present study, we intended to investigate protective effects of quercetin on mitochondria, ATP synthesis and complex I mRNA expression with a view to develop a model to study mitochondrial biogenesis and to identify chemicals of plant origin that cause mitochondrial biogenesis. We demonstrate that quercetin shows protective effects on oxidative stress induced myocyte cell death through increasing ATP synthesisan indirect but ultimate indicator of mitochondrial biogenesis. An understanding of the mechanisms underlying mitochondrial biogenesis may facilitate the development of therapeutics in diseases involving mitochondrial dysfunction.

\section{Materials and Method}

\section{Materials}

Quercetin, Antimycin-A and DMSO were obtained from Sigma Chemical Co (St Louis, MO). PenStrep and DMEM medium were obtained from VWR international (Randor, PA), FBS was obtained from ATCC (Manassas, VA), MitoSOXTM Red Mitochondrial super oxide indicator was obtained from Thermofisher (Grand Island, NY). Trypsin-EDTA was purchased from Invitrogen (Carlsbad, CA). CytoTox-ONE Homogenous Membrane Integrity Assay kit and CellTiter-Glo luminescent cell viability assay kit (ATP) were obtained from Promega (Madison, WI). The BCA protein assay kit was purchased from Pierce (Rockford, IL). All other reagents were of analytical grade and were purchased from commercially available vendors.

\section{Cell culture}

Rat L6 were obtained from ATCC and expanded in laboratory. Cells were grown in DMEM media with glutamate, supplemented with $10 \%$ FBS and antibiotics (PenStrep). For fluorescence and for luminescence assays that involved a microplate reader, the cells were seeded in flat micro-clear bottom 96-well plates (BD Falcon, BD Biosciences, San Jose, CA) at a density of $3 \times 10^{5} / \mathrm{mL}$. All drugs were either directly added to the culture medium or dissolved in dimethyl sulfoxide (DMSO) and then added to the culture medium (final DMSO concentration was $0.1 \% \mathrm{v} / \mathrm{v}$ ). Plates were incubated at $5 \% \mathrm{CO}_{2}, 37^{\circ} \mathrm{C}$ and $95 \%$ relative humidity for 24 or $48 \mathrm{~h}$ time period.

\section{Determination of cell injury}

Release of lactate dehydrogenase (LDH) into the extracellular medium was used as an indicator of cytotoxicity. The relative activity of $\mathrm{LDH}$ in the medium (as percentage of total intracellular plus extracellular activity) was determined using a CytoTox-ONE Homogenous Membrane Integrity Assay kit. In the presence of LDH, resazurin is converted to resorufin which was quantified in a microplate reader (excitation $560 \mathrm{~nm}$, emission $590 \mathrm{~nm}$ ). Enzyme activity in the medium was sampled at indicated time points and expressed as percentage of total intracellular and extracellular LDH activity.

\section{Determination of cellular ATP content}

For determination of ATP content we used a CellTiter-Glo Luminescent cell viability kit. Luciferin is mono-oxygenated in the presence of ATP, $\mathrm{Mg}^{2+}$, and molecular oxygen to luminescent compound oxyluciferin. Briefly, after treatment and incubation with test chemicals, cells were treated with premixed CellTiter-Glo reagent, then plates were incubated for $10 \mathrm{~min}$ at room temperature to stabilize luminescent signal. Luminescence emitted out by oxyluciferin is then measured with luminometer.

\section{Determination of cell number}

Measurement of monolayer protein content and actual cell count over time were used to estimate cell number. Cells were seeded in 6well plates at a concentration of $1.2 \times 10^{6}$ cells/well. Cells were treated with either $0.1 \% \mathrm{v} / \mathrm{v}$ DMSO or $50 \mu \mathrm{M}$ quercetin and incubated for $24 \mathrm{~h}$ time period at $5 \% \mathrm{CO}_{2}, 37^{\circ} \mathrm{C}$ and $95 \%$ relative humidity. To determine protein concertation L6 myocyte monolayers were washed with PBS solubilized in Triton buffer (0.05\% Triton X-100, $100 \mathrm{mM}$ Tris-base, and $150 \mathrm{mM} \mathrm{NaCl}, \mathrm{pH}$ 7.5). Protein concentrations, were determined by the bicinchoninic acid (BCA) method according to the manufacturer's instructions. Actual cell count was made by harvesting cells and counting using phase contrast microscope and Neubauer chamber.

\section{Measurement of mitochondrial superoxide}

Mitochondrial superoxide was measured with the cell-permeable fluorogenic probe, hydroethidine coupled to triphenylphosphonium (mito-HE, MitoSox Red ${ }^{\star}$ ). Being a lipophilic cation, MitoSOX Red is selectively targeted to mitochondria where it specifically reacts with superoxide anion. Quercetin, AMA or quercetin and AMA preexposed cells were loaded with MitoSOX Red $(1 \mu \mathrm{M})$ for $10 \mathrm{~min}$ at $37^{\circ} \mathrm{C}$, washed with PBS, and then fluorescence was determined using the excitation at $396 \mathrm{~nm}$ and emission at $580 \mathrm{~nm}$ as described in Kashimshetty et al. [19]. Relative Fluorescence Units (RFUs) of untreated cells was considered as 100 percent.

\section{Quantitative real-time PCR}

NADH dehydrogenase (ubiquinone) 1 beta subcomplex subunit 8 (NDUFB8) is also known as complex I, is located in the mitochondrial inner membrane. It is encoded by nuclear DNA and catalyzes the oxidation of NADH and electron transfer. Relative increase in levels of expression of NDUFB8 correlates with increased mitochondrial function [20-22]. For NDUFB8 mRNA expression, total RNA was isolated from L6 cells with RNeasy Mini Kit (Qiagen, Valencia, CA). cDNA was synthesized from $1 \mu \mathrm{g}$ of RNA template using a RevertAid ${ }^{\text {ix }}$ First Strand cDNA Synthesis Kit (Thermo Fisher Scientific, Grand island, NY). PCRs were carried out by using $3 \mu$ of 1:3 diluted cDNA template combined with Maxima ${ }^{\oplus}$ SYBR Green qPCR Master Mix (2X) at a final concentration of $1 \mathrm{X}$ (Thermo Fisher Scientific, Grand Island, NY.), ROX (Thermo Fischer Scientific, Grand Island, NY.) and primers (Thermo Fisher Scientific, Grand Island, NY) at a concentration of 10 $\mathrm{nM}$ and $400 \mathrm{nM}$ respectively. For real-time PCRs primer sequences for NDUFB8 were: FW: 5'-GGC GAT CCC AAC AAA GAA CC-3'; REV: 5'-TTT CTA GGA TTG AAG GAG TC-3' and the abundance of mRNAs was normalized against tubulin FW: 5'-CTC TCT GTC GAT TAC GGC AAG-3'; REV: 5'-TGG TGA GGA TGG AGT TGT AGG-3' using the $\Delta \Delta C t$ method. Relative change in mRNA expression was then expressed as fold change over untreated cells.

\section{Statistical analysis}

All data were expressed as mean \pm SEM of at least three independent experiments. Each experiment is performed in triplicates 
$(\mathrm{N}=3)$. Statistical analysis was performed using SPSS 20.0 (SPSS, Chicago, IL) by using one-way ANOVA followed by Tukey's post hoc test. Data were considered statistically significant at $P \leq 0.05$.

\section{Results}

\section{Effects of quercetin on antimycin-A (AMA) induced toxicity}

Cell viabilities were determined using L6 cells and LDH assay for various concentrations of quercetin. Dose dependent cytotoxicity was observed with quercetin $(0-500 \mu \mathrm{M})$ for $24 \mathrm{~h}$ and $48 \mathrm{~h}$ time points. Significant difference in \% LDH leakage has been noted at quercetin concentrations 100,250 and $500 \mu \mathrm{M}$ ) at $24 \mathrm{~h}$ time point and for 50, 100,250 and $500 \mu \mathrm{M}$ concentrations at $48 \mathrm{~h}$ time point (Figure 1A).

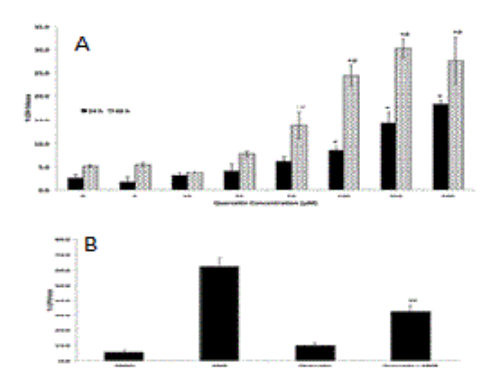

Figure 1: A. Effects of quercetin on cell viability and cytoprotective effect of quercetin on L6 cells treated with AMA. A. Effects of quercetin on cell viability at various concentrations and time points. B. Cytoprotective effect of quercetin on L6 cells treated with AMA. L6 cells were pretreated with quercetin $50 \mu \mathrm{M}$ for $1 \mathrm{~h}$ and then treated with $100 \mu \mathrm{g} / \mathrm{mL}$ of AMA. Significant differences over vehicle control denoted as ${ }^{*} \mathrm{p} \leq 0.05$. Significant differences over same treatment group but different time points denoted as \# $\mathrm{p} \leq 0.05$.

We used Antimycin-A to induce cell death in myocyte through mitochondrial dysfunction. We then determined the dose and time of exposure to $100 \mu \mathrm{g} / \mathrm{mL}$ AMA required to reduce cell viability by $50 \%$ after $24 \mathrm{~h}$ incubation (data not shown). Cells were pretreated with quercetin $50 \mu \mathrm{M}$ concertation that showed no significant effects on myocyte cell death at $24 \mathrm{~h}$ time period. One hour $(1 \mathrm{~h})$ after quercetin treatment, cells were then treated with $100 \mu \mathrm{g} / \mathrm{mL}$ AMA in the presence of quercetin. Significant reduction in cytotoxicity $(\sim 30 \%)$ has been noted in group treated with quercetin $(50 \mu \mathrm{M})+\mathrm{AMA}$ compared with AMA alone (Figure 1B).

\section{Effects of quercetin on ATP levels}

To determine whether the quercetin affected energy production, we measured ATP levels. Cellular ATP levels after treatment with test chemicals were determined on L6 cells by CellTiter-Glo Luminescent cell viability kit. Significant difference in \% ATP has been noted at quercetin concentrations $500 \mu \mathrm{M}$ at $24 \mathrm{~h}$ and 250 and $500 \mu \mathrm{M}$ at $48 \mathrm{~h}$ time point (Figure 2A). Non-significant increase in ATP levels has been noted with concentration 5,10 and $25 \mu \mathrm{M}$ at $48 \mathrm{~h}$ time point as compared to $24 \mathrm{~h}$ time point. To determine protective effects of quercetin on AMA-induced ATP loss, cells were then pretreated with quercetin $50 \mu \mathrm{M}$ and then $1 \mathrm{~h}$ after quercetin treatment cells were treated with $100 \mu \mathrm{g} / \mathrm{mL}$ AMA in the presence of quercetin. Quercetin
$(50 \mu \mathrm{M})$ increased cellular ATP by $18 \%$ compared to AMA alone (Figure 2B).

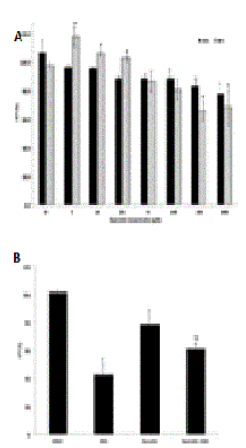

Figure 2: A. Effects of quercetin on total cellular ATP levels. A. Total ATP levels in L6 cells treated with various concentration of quercetin at different time points. B. Total ATP levels in L6 cells treated with AMA. Cells were pretreated with quercetin $50 \mu \mathrm{M}$ for 1 $\mathrm{h}$ then treated with $100 \mu \mathrm{g} / \mathrm{mL}$ of AMA. ATP levels were measured using the ATP Lite luminescence-based assay in which results are reported as a percentage of luminescence units over untreated cells. Significant differences over vehicle control denoted as ${ }^{*} \mathrm{p} \leq 0.05$. Significant differences over same treatment group but different time points denoted as $\# \mathrm{p} \leq 0.05$.

\section{Effects of quercetin on myocyte cell number}

Mitochondrial biogenesis has been identified as a compensatory response to cellular injury and often increased mitochondrial biogenesis attributed to increased cell proliferation. To determine whether cell number changed during the $24 \mathrm{~h}$ exposure to quercetin, the amount of cellular protein per well, a marker of cell number was measured in the presence and absence of quercetin $(50 \mu \mathrm{M})$ after $24 \mathrm{~h}$ treatment. Monolayer protein content as well as cell number did not change in the presence of quercetin (Figures $3 \mathrm{~A}$ and $3 \mathrm{~B}$ ).

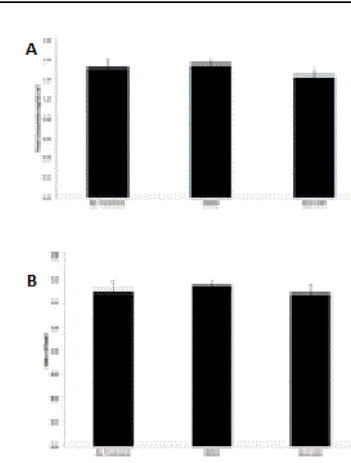

Figure 3: Effects of quercetin on rat L6 cell number. A. Cells were treated with quercetin $50 \mu \mathrm{M}$. Protein content per well was determined using BCA protein assay kit after $24 \mathrm{~h}$ treatment with quercetin. B. Total cell count Cells were counted using neubauer's chamber and trypan blue after $24 \mathrm{~h}$ treatment with quercetin. 


\section{Changes in mitochondrial superoxide levels}

Quantitative measurements of mean intensity from the AMAinduced cells demonstrated a $187.8 \%$ increase compared with u cells (Figure 4).

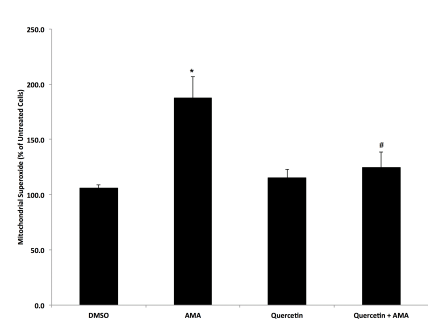

Figure 4: Determination of mitochondrial superoxide production measured using MitoSOX Red. L6 cells were pretreated with quercetin $50 \mu \mathrm{M}$ for $1 \mathrm{~h}$, and then $100 \mu \mathrm{g} / \mathrm{mL}$ AMA was added. Quercetin, AMA or quercetin and AMA preexposed cells were loaded with MitoSOX Red $(1 \mu \mathrm{M})$ for 10 min at $37^{\circ} \mathrm{C}$, washed with PBS, and the fluorescence was determined using the excitation at $396 \mathrm{~nm}$ and emission at $580 \mathrm{~nm}$ Relative Fluorescence Units (RFUs) of untreated cells were considered as 100 percent. Significant differences over vehicle control denoted as ${ }^{*} \mathrm{p} \leq 0.05$. Significant differences over same treatment group but different time point denoted as \# $\mathrm{p} \leq 0.05$.

In contrast, mitochondrial superoxide levels were $115.5 \%$ when cells were treated with quercetin indicating lower mitochondrial superoxide levels. In the presence of quercetin+AMA, mitochondrial super oxide levels were $124.5 \%$ suggesting protective effect of quercetin on mitochondrial superoxide production.

\section{NDUFB8 mRNA expression}

We also examined electron transport chain (ETC) integrity by examining mRNA levels of NDUFB8 which is a nuclear-encoded ETC protein. We found significant increase in NDUFB8 mRNA expression in Quercetin+AMA treated cells over cells treated with AMA alone (Figure 5).

\section{Discussion}

Mitochondrial dysfunction plays an important role in cellular injury, recovery after tissue damage, and pathogenesis of various disorders and conditions such as Alzheimer's, aging, development of insulin resistance in diabetes etc. Preventing mitochondrial dysfunction by increasing mitochondrial biogenesis is important in reducing cellular damage. In the present study, we focused on the flavonoid quercetin for its effect on prevention of mitochondrial dysfunction. We used AMA to induce mitochondrial dysfunction and to induce cytotoxicity. In our study, cell viability was increased by the addition of quercetin in AMA treated cells over just AMA treated cells. Hese results agree with the findings of others that quercetin protects against toxicant-induced cell damage [14,23-26]. Mitochondrial dysfunction induced by AMA results in decreased ATP production. Oxidative stress increases respiration and generation of ROS, resulting in ATP depletion [27]. AMA causes oxidative stress and mitochondrial dysfunction by binding to the matrix side of complex III and inhibiting the mitochondrial electron transport system [28]. We show here that treatment with quercetin in AMA exposed cells significantl prevented loss of ATP, suggesting that quercetin protect cells from mitochondrial dysfunction

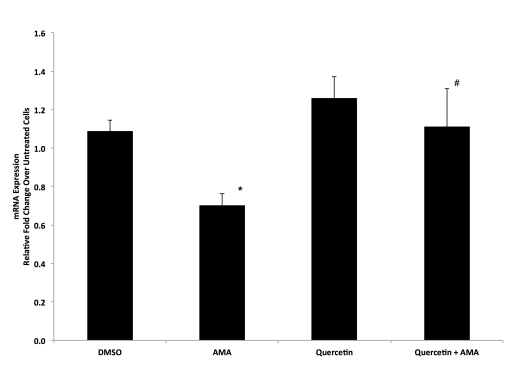

Figure 5: NDUFB8 mRNA expression analysis in rat L6 cells. Total RNA was isolated from L6 cells, pretreated with quercetin for $1 \mathrm{~h}$, and then exposed to AMA and quercetin. $1 \mu \mathrm{g}$ of total RNA converted to cDNA. Using primer against NDUFB8, relative intensity of cDNA was detected with SYBR Green qPCR. Data were expressed as relative fold change over untreated cells. Significant differences over vehicle control denoted as ${ }^{*} \mathrm{p} \leq 0.05$. Significant differences over same treatment group but different time point denoted as \# $\mathrm{p} \leq 0.05$.

Mitochondrial ROS production is intimately linked to mitochondrial dysfunction, aging, insulin resistance, exercise tolerances, etc. [29]. Our data presented here show that cells treated with quercetin exhibited decreased mitochondrial super oxide production. The MitoSOX Red results indicate that mitochondrial superoxide was increased in AMA-induced cells. Cells treated with quercetin prevented AMA-induced superoxide production by mitochondria. NDUFB8 is a nuclear encoded protein of electron transport chain. In the present study we demonstrated that AMA significantly reduced NDUFB8 expression. On the other hand cell pretreated with quercetin and then treated with AMA showed increased NDUFB8 expression without causing appreciable increase in cell number implying improved mitochondrial function as evidenced from increased ATP content. Further newly synthesized or divided mitochondria works efficiently as quercetin+AMA treated cells showed comparatively lower super oxide production over cells treated with AMA alone. Mitochondrial dysfunction, characterized by a decline in cellular ATP, cytotoxicity, and superoxide generation is central to the execution of myocyte cell death and thus increasing glucose metabolism in muscle cells to effectively burning glucose to ATP in mitochondria without generating overtly more superoxide is important. Therapeutic strategies to increase mitochondrial biogenesis and reduce oxidative stress would therefore be useful in diseases that are associated with mitochondrial dysfunction.

\section{Conclusion}

In conclusion, quercetin protects AMA-induced cell death. These results suggest that the protection of cell death is a result of increased mitochondrial biogenesis as evidenced from improved mitochondrial function through reduced oxidative stress, increased ATP levels and increased nuclear encoded NDUFB8 mRNA expression. Rat L6 myocytes in conjunction with direct markers of mitochondrial biogenesis, and with further understanding of mitochondrial biogenesis will be an useful tool to screen and identify new chemicals, 
particularly of plant origin that cause mitochondrial biogenesis. The identification of novel molecules based on mitochondrial biogenesis principle may play an important role in the treatment and prevention of diseases associated with mitochondrial dysfunction.

\section{Acknowledgement}

Funding for this project was provided by Roseman University of Health Sciences.

\section{References}

1. Li L, Pan R, Li R, Niemann B, Aurich A, et al. (2011) Mitochondria biogenesis and peroxisome proliferator-activated receptor-gamma coactivator-1alpha (PGC-1alpha) deacetylation by physical activity: intact adipocytokine signaling is required. Diabetes 60: 157-167.

2. Jorgensen SB, Richter EA, Wojtaszewski JF (2006) Role of AMPK in skeletal muscle metabolic regulation and adaptation in relation to exercise J Physiol. 574: 17-31.

3. Liu J, Shen W, Zhao B, Wang Y, Wertz K, et al. (2009) Targeting mitochondrial biogenesis for preventing and treating insulin resistance in diabetes and obesity: Hope from natural mitochondrial nutrients. Adv Drug Deliv Rev 61: 1343-1352.

4. Jornayvaz, FR Shulman GI (2010) Regulation of mitochondrial biogenesis. Essays Biochem 47: 69-84.

5. Suwa M, Egashira T, Nakano H, Sasaki H, Kumagai S, et al. (2006) Metformin increases the PGC-1alpha protein and oxidative enzyme activities possibly via AMPK phosphorylation in skeletal muscle in vivo. J Appl Physiol 101: 1685-1692.

6. Westermann B (2010) Mitochondrial fusion and fission in cell life and death. Nat Rev Mol Cell Biol 11: 872-884.

7. Civitarese AE, Carling S, Heilbronn LK, Hulver MH, Ukropcova B, et al. (2007) Calorie restriction increases muscle mitochondrial biogenesis in healthy humans. PLoS Med 4: e76.

8. Landete JM (2012) Updated knowledge about polyphenols: functions, bioavailability, metabolism, and health. Crit Rev Food Sci Nutr 52: 936-948.

9. Beecher GR (2003) Overview of dietary flavonoids: nomenclature occurrence and intake. J Nutr 133: 3248S-3254S.

10. Middleton E, Kandaswami C, Theoharides TC (2000) The effects of plant flavonoids on mammalian cells: implications for inflammation, heart disease, and cancer. Pharmacol Rev 52: 673-751.

11. Min YD, Choi CH, Bark H, Son HY, Park HH, et al. (2007) Quercetin inhibits expression of inflammatory cytokines through attenuation of NFkappaB and p38 MAPK in HMC-1 human mast cell line. Inflamm Res 56: 210-215.

12. Rezaei SR, Eidi A, Zarghami N, Barzegar A (2014) Intracellular ROS protection efficiency and free radical-scavenging activity of quercetin and quercetin-encapsulated liposomes. Artif Cells Nanomed Biotechnol 44: $1-7$.

13. Davis JM, Murphy EA, Carmichael MD (2009) Effects of the dietary flavonoid quercetin upon performance and health. Curr Sports Med Rep 8: 206-213.
14. Davis JM, Murphy EA, Carmichael MD (2009) Quercetin increases brain and muscle mitochondrial biogenesis and exercise tolerance. Am J Physiol Regul Integr Comp Physiol 296: R1071-1077.

15. Lenaz G, Bovina C, D'Aurelio M, Fato R, Formiggini G, et al. (2002) Role of mitochondria in oxidative stress and aging. Ann N Y Acad Sci 959: 199-213.

16. Baum H, Rieske JS, Silman HI, Lipton SH (1967) On the mechanism of electron transfer in complex iii of the electron transfer chain. Proc Natl Acad Sci 57: 798-805.

17. Rieske JS, Lipton SH, Baum H, Silman HI (1967) Factors affecting the binding of antimycin A to complex 3 of the mitochondrial respiratory chain. J Biol Chem 242: 4888-4896.

18. Drose S, Brandt U (2008) The mechanism of mitochondrial superoxide production by the cytochrome bc1 complex. J Biol Chem 283: 21649-21654.

19. Kashimshetty R, Desai VG, Kale VM, Lee T, Moland CL, et al. (2009) Underlying mitochondrial dysfunction triggers flutamide-induced oxidative liver injury in a mouse model of idiosyncratic drug toxicity. Toxicol Appl Pharmacol 238: 150-159.

20. Janssen RJ, Nijtmans LG, van den Heuvel LP, Smeitink JA (2006) Mitochondrial complex I: structure, function and pathology. J Inherit Metab Dis 29: 499-515.

21. Lazarou M, Smith SM, Thorburn DR, Ryan MT, McKenzie M (2009) Assembly of nuclear DNA-encoded subunits into mitochondrial complex $\mathrm{IV}$, and their preferential integration into supercomplex forms in patient mitochondria. FEBS J 276: 6701-6713.

22. Lazarou M, Thorburn DR, Ryan MT, McKenzie M (2009) Assembly of mitochondrial complex I and defects in disease. Biochim Biophys Acta 1793: 78-88

23. Liu CM, Ma JQ, Sun YZ (2010) Quercetin protects the rat kidney against oxidative stress-mediated DNA damage and apoptosis induced by lead. Environ Toxicol Pharmacol 30: 264-271.

24. Liu CM, Zheng YL, Lu J, Zhang ZF, Fan SH, et al. (2010) Quercetin protects rat liver against lead-induced oxidative stress and apoptosis. Environ Toxicol Pharmacol 29: 158-166.

25. Park C, So HS, Shin CH, Baek SH, Moon BS, et al. (2003) Quercetin protects the hydrogen peroxide-induced apoptosis via inhibition of mitochondrial dysfunction in $\mathrm{H} 9 \mathrm{c} 2$ cardiomyoblast cells. Biochem Pharmacol 66: 1287-1295.

26. Renugadevi J, Prabu SM (2010) Quercetin protects against oxidative stress-related renal dysfunction by cadmium in rats. Exp Toxicol Pathol 62: 471-481.

27. Tiwari BS, Belenghi B, Levine A (2002) Oxidative stress increased respiration and generation of reactive oxygen species, resulting in ATP depletion, opening of mitochondrial permeability transition, and programmed cell death. Plant Physiol 128: 1271-1281.

28. Chen Q, Vazquez EJ, Moghaddas S, Hoppel CL, Lesnefsky EJ (2003) Production of reactive oxygen species by mitochondria: central role of complex III. J Biol Chem 278: 36027-36031.

29. Fujisawa K, Nishikawa T, Kukidome D, Imoto K, Yamashiro T, et al. (2009) TZDs reduce mitochondrial ROS production and enhance mitochondrial biogenesis. Biochem Biophys Res Commun 379: 43-48. 